\title{
A Case Report of Adult-Onset Alexander Disease with a Tumor-Like Lesion in the Lateral Ventricle
}

\author{
Tongjia Cai ${ }^{a}$ Sisi Jing ${ }^{a} \quad$ Ying $\mathrm{Li}^{\mathrm{a}}$ Jianjun $\mathrm{Wu}^{\mathrm{a}}{ }^{\mathrm{b}}$ \\ aDepartment of Neurology, Jing'an District Centre Hospital of Shanghai, Shanghai, China; \\ bepartment of Neurology, Huashan Hospital, Shanghai Medical College, Fudan University, \\ Shanghai, China
}

\section{Keywords}

Alexander disease $\cdot$ Adult-onset $\cdot$ c.236G $>$ A $\cdot$ Tumor-like lesion

\begin{abstract}
Adult-onset Alexander disease (AOAD) is an autosomal dominant progressive astrogliopathy caused by pathogenic variants in glial fibrillary acidic protein (GFAP). Individuals with this disorder often present with a typical neuroradiologic pattern, including frontal white matter abnormality with contrast enhancement, atrophy and signal intensity changes of the medulla oblongata and upper cervical cord on MRI. Focal lesions are rarely seen in AOAD, which causes concern for primary malignancies. This study aimed to present the case of a 37-year-old male patient initially diagnosed with an astrocytoma in the lateral ventricle that was later identified as GFAP mutation-confirmed AOAD. GFAP sequencing revealed a heterogeneous missense mutation point c. $236 \mathrm{G}>\mathrm{A}$. Hence, AOAD should be considered in patients with tumor-like lesion brain lesion in association with atrophy of medulla oblongata and upper cervical spinal cord, and frontal white matter abnormality with contrast enhancement.
\end{abstract}

(c) 2021 The Author(s).

Published by S. Karger AG, Basel

\section{Introduction}

Alexander disease (AD) is a progressive degenerative leukodystrophy dominantly caused by mutations in the glial fibrillary acidic protein (GFAP) gene on chromosome 17q21 [1, 2], and a disorder of astroglia characterized histopathologically by the presence of Rosenthal fibers on hematoxylin and eosin staining [3]. This study aimed to present a case of a 37-year-old male patient initially diagnosed with an astrocytoma in the lateral ventricle that was later identified as Adult-onset AD (AOAD). 

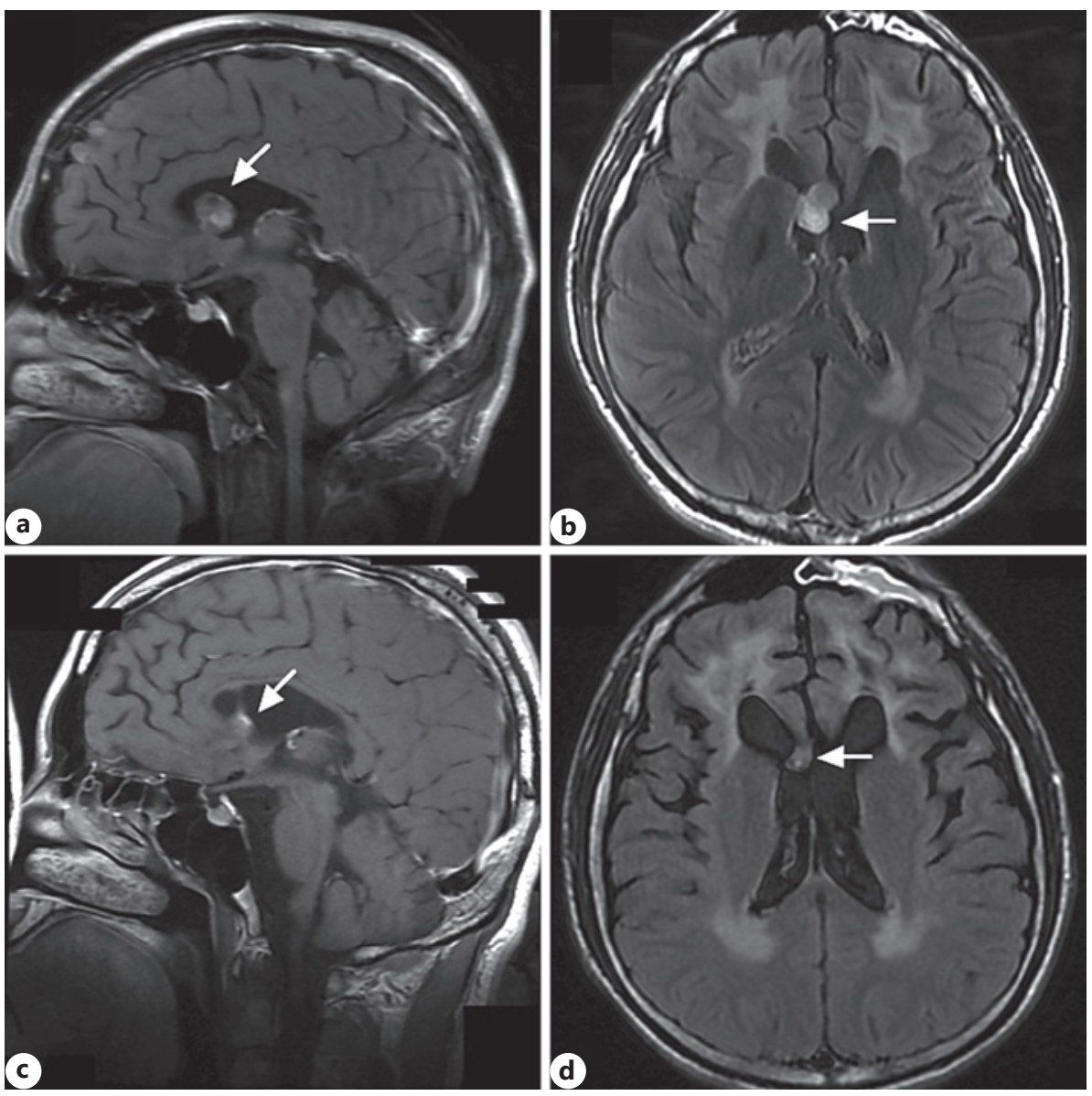

Fig. 1. a Sagittal cranial MRI showed that the tumor-like lesion (arrowhead) in the lateral ventricle was hyper-hyperintense in the T2 FLAIR with gadolinium enhancement image. b Sagittal cranial MRI also showed atrophy in the upper cervical spinal. Axial cranial MRI showed that the tumor-like lesion (arrowhead) in the lateral ventricle was hyper-hyperintense in the T2 FLAIR with gadolinium enhancement image. c Sagittal cranial MRI showed that the tumor-like lesion (arrowhead) in the lateral ventricle shrunk in the T2 FLAIR with gadolinium enhancement image after stereotactic radiosurgery therapy. d Axial cranial MRI showed that the tumor-like lesion (arrowhead) in the lateral ventricle shrunk in the T2 FLAIR with gadolinium enhancement image after stereotactic radiosurgery therapy. FLAIR, fluid attenuated inversion recovery.

\section{Case Presentation}

A 37-year-old male patient was evaluated at our institution with an 18-month history of progressive neurological symptom. His past medical history was normal. At the age of 35 years, he developed weakness in his right limbs, especially the right lower extremity. MRI of the brain demonstrated that a tumor-like lesion was $16 \times 12 \times 17 \mathrm{~mm}^{3}$ in the lateral ventricle (shown in Fig. 1a, b, arrowhead). The tumor-like lesion was misdiagnosed as astrocytoma at another hospital 4 months after onset. The tumor-like lesion in the lateral ventricle shrunk (shown in Fig. 1c, d, arrowhead) after stereotactic radiosurgery therapy, but the symptoms did not improve. Neurological examination revealed horizontal nystagmus of both eyes. The pharyngeal reflex was absent, motor strength in the neck was of grade $3 / 5$, right upper limb muscle strength was of grade $4 / 5$, right lower limb muscle strength was of grade $3 / 5$, and left-limb muscle strength was of grade $4 / 5$. Moreover, deep tendon reflexes 
were brisk in the upper and lower limbs. Bilateral Babinski sign was present. The sensation was intact.

Repeat MRI showed hyperintensity involving the midbrain, medulla oblongata, cervical spinal cord (shown in Fig. 2a-d, arrowhead), and the cerebral white matter abnormalities with frontal predominance (shown in Fig. 2e, arrowhead). Repeat MRI also showed mild atrophy in the midbrain, medulla oblongata, upper cervical spinal cord in sagittal T2WI (shown in Fig. 2f), and hyperintensity involving the corticospinal tracts extending from carotid 3 (shown in Fig. $2 \mathrm{~g}$, arrowhead) to carotid 7 (shown in Fig. 2h, arrowhead) in the T2WI image. The laboratory tests were normal.

Whole-exome sequencing for him and his parents was completed. It revealed heterogeneous missense mutation point in c.236G >A (guanine > adenine) in exon 1 of the GFAP gene, leading to amino acid change in p.R79H (arginine > histidine) (shown in Fig. 3a-c), confirming the diagnosis of AOAD.

\section{Discussion}

$\mathrm{AD}$ has traditionally been categorized by symptom onset: infantile (birth to 2 years), juvenile (2-14 years), and adult ( $>14$ years) [4]. The MRI findings of AD included frontal predominance of white matter abnormalities, brainstem abnormalities, and contrast enhancement in the periventricular region, ventricular lining, optic chiasm, and brainstem. The patient in our study should be categorized into AOAD. In AOAD, frontal white matter abnormality with contrast enhancement [5], atrophy and signal intensity changes of the medulla oblongata and upper cervical cord on MRI have been reported as specific features [6]. Based on neurological symptoms and MRI findings, AD is classified by Yoshida et al. [7] into three subtypes: cerebral AD (type I), bulbospinal AD (type II), and intermediate form (type III). The patient in the present study had symptoms consistent with intermediate form (type III) based on his MRI findings and clinical course, similar to a case reported earlier by Nam et al. [5].

$\mathrm{AD}$ had presented in infantile and juvenile patients as a mass lesion, involving, for example, the brainstem or optic chiasma. These lesions contain large numbers of Rosenthal fiber [8]. However, tumor-like lesion in the lateral ventricle is a rare condition in AOAD. Poppel et al. [9] reported a juvenile-onset case of AD with a focal brainstem lesion, which was presumed to be neoplastic. Several histologic features of AD can mimic brain tumors. Rosenthal fiber, a pathologic hallmark of $\mathrm{AD}$, formation in the astrocytes of the brain and the spinal cord, is also seen in several low-grade primary brain tumors (e.g., pilocytic astrocytoma, ganglioglioma, and pleomorphic xanthoastrocytoma). Cytologically atypical astrocytes, a recognized feature of $\mathrm{AD}$, can also lead to the misdiagnosis of pilocytic astrocytoma/ganglioglioma and unnecessary invasive interventions [10].

Although GFAP which is part of the component of Rosenthal fibers is particularly expressed in neural stem cells in subventricular zone of the lateral ventricles [11], a tumor-like lesion in the lateral ventricle is a rare condition in AOAD. It is important for clinicians to be aware that tumor-like lesions can occur during the course of AOAD, to avoid delayed diagnosis or misdiagnosis which may lead to unnecessary procedures and inappropriate treatment. We suggest that, Clinicians should be aware of AOAD and consider genetic testing for GFAP gene mutation in patients presenting with atypical tumor-like focal lesions in association with atrophy of medulla oblongata and upper cervical spinal cord, and frontal predominance of white matter abnormalities.

The authors thank the patient and his family for participating in this research. The authors are thankful to Fudan University for developing and encouraging the research platform to serve the community and world.

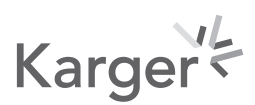



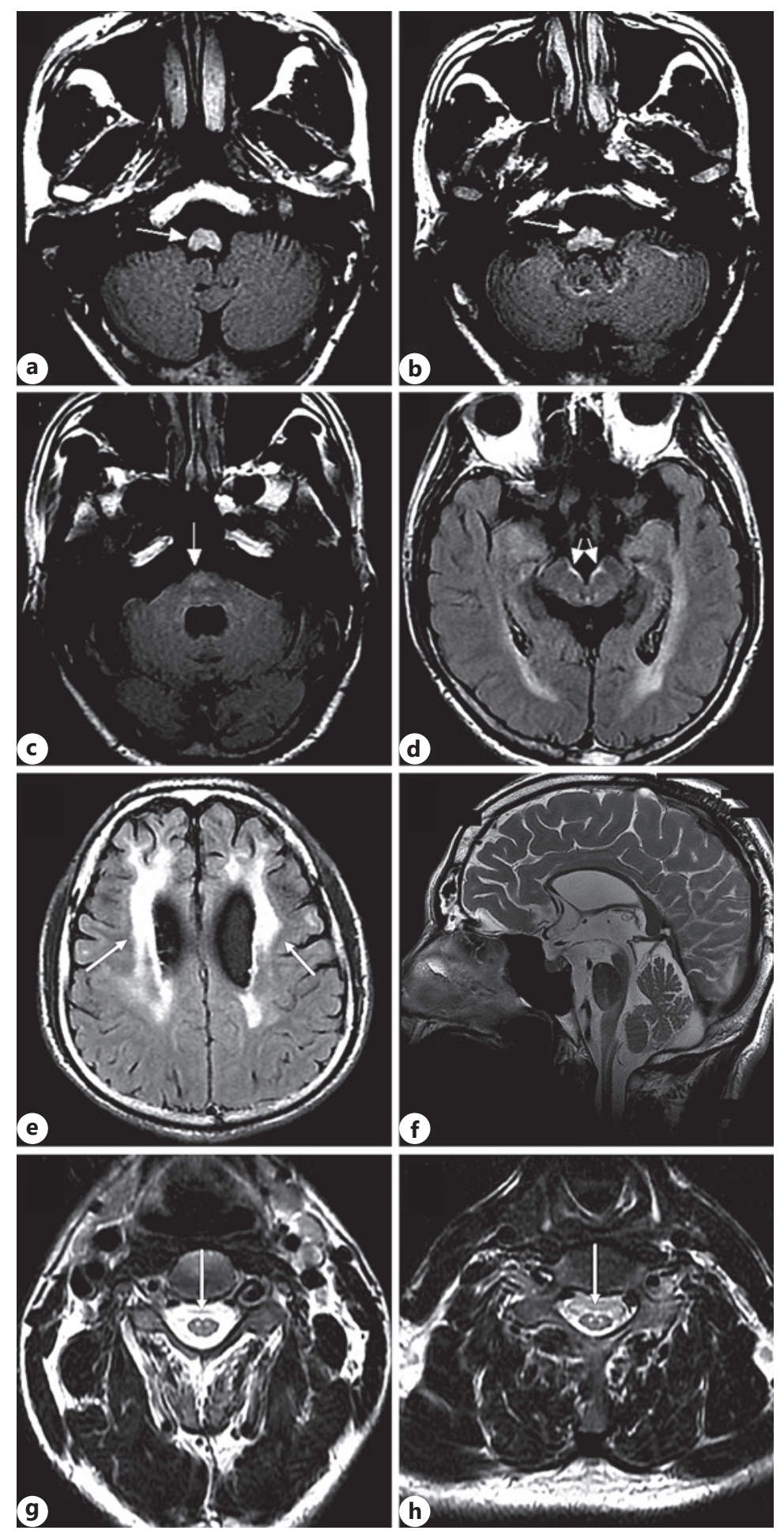

Fig. 2. Axial T2 FLAIR images showing hyperintensity in the cervical spinal cord (a, arrowhead), medulla oblongata (b, c, arrowheads), and midbrain (d, arrowhead). Cranial MRI showed cerebral white matter abnormalities with frontal predominance (arrowhead) in axial T2 FLAIR images (e). Cranial MRI showed mild atrophy in the midbrain, medulla oblongata, and upper cervical spinal cord in the sagittal T2WI image (f). Axial spinal MRI showed hyperintensity involving the corticospinal tracts extending from carotid 3 (g, arrowhead) to carotid 7 (h, arrowhead) in T2WI. FLAIR, fluid attenuated inversion recovery. 
Fig. 3. Chromatograms of sequencing results of the patient (a), father (b), and mother (c). The area of the GFAP gene exon 1 had a heterogeneous missense mutation point c.236G>A (guanine $>$ adenine), leading to amino acid change in p.R79H (arginine > histidine). GFAP, glial fibrillary acidic protein.

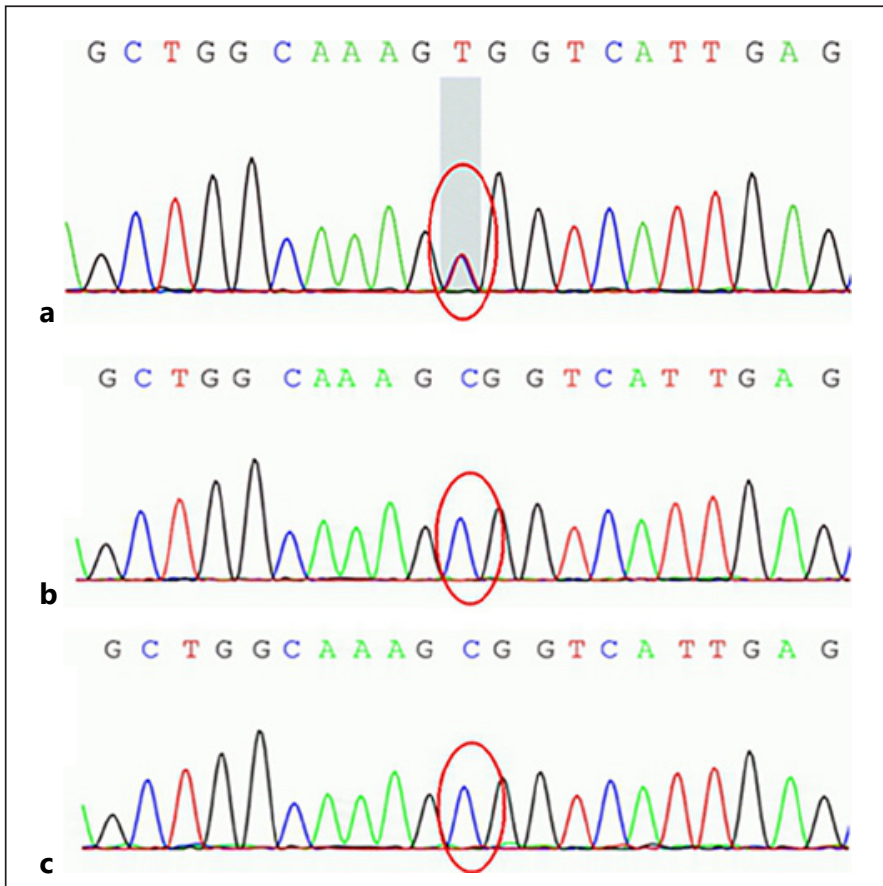

\section{Statement of Ethics}

Written informed consent was obtained from the parent/guardian of the patient for the publication of this report (including images). The research in this paper was conducted ethically in accordance with the World Medical Association Declaration of Helsinki.

\section{Conflict of Interest Statement}

The authors have no conflicts of interest to declare.

\section{Funding Sources}

This work was supported by financial grants from the Joint Medical Research Program of Shanghai Jing'an District Science and Technology Commission and Health Commission (No. 2018MS01).

\section{Author Contributions}

T.C. contributed to the manuscript development. All the authors were equally involved in the medical management of the patient and approved final version of the manuscript. 


\section{Case Reports in Neurology}

\begin{tabular}{l|l}
\hline Case Rep Neurol 2021;13:355-360 \\
\hline DOI: 10.1159/000516256 & $\begin{array}{l}\text { @ 2021 The Author(s). Published by S. Karger AG, Basel } \\
\text { www.karger.com/crn }\end{array}$ \\
\hline
\end{tabular}

Cai et al.: Adult-Onset Alexander Disease with a Tumor-like Lesion

\section{References}

1 Li R, Johnson AB, Salomons G, Goldman JE, Naidu S, Quinlan R, et al. Glial fibrillary acidic protein mutations in infantile, juvenile, and adult forms of Alexander disease. Ann Neurol. 2005;57:310-26.

2 Tavasoli A, Armangue T, Ho CY, Whitehead M, Bornhorst M, Rhee J, et al. Alexander disease: a leukodystrophy that may mimic brain tumor. J Child Neurol. 2017;32(2):184-7.

3 Chang KE, Pratt D, Mishra BB, Edwards N, Hallett M, Ray-Chaudhury A. Type II (adult onset) Alexander disease in a paraplegic male with a rare D128N mutation in the GFAP gene. Clin Neuropathol. 2015;34(5):298-301.

4 Prust M, Wang J, Morizono H, Messing A, Brenner M, Gordon E, et al. GFAP mutations, age at onset, and clinical subtypes in Alexander disease. Neurology. 2011;77(13):1287-94.

5 Nam TS, Kang KW, Choi SY, Kim MK. Teaching NeuroImages: Alexander disease with features of both frontal and bulbospinal involvement. Neurology. 2018;91(4):e396-7.

6 Yonezu T, Ito S, Kanai K, Masuda S, Shibuya K, Kuwabara S. A case of adult-onset alexander disease featuring severe atrophy of the medulla oblongata and upper cervical cord on magnetic resonance imaging. Case Rep Neurol. 2012;4(3):202-6.

7 Yoshida T, Sasaki M, Yoshida M, Namekawa M, Okamoto Y, Tsujino S, et al. Nationwide survey of Alexander disease in Japan and proposed new guidelines for diagnosis. J Neurol. 2011;258:1998-2008.

8 Sosunov A, Olabarria M, Goldman JE. Alexander disease: an astrocytopathy that produces a leukodystrophy. Brain Pathol. 2018;28:388-98.

9 Poppel KV, Ellison D, Broniscer A, Patay Z. Alexander disease: an important mimicker of focal brainstem glioma. Pediatr Blood Cancer. 2010;53(7):1355-6.

10 Machol K, Jankovic J, Vijayakumar D, Burrage LC, Jain M, Lewis RA, et al. Atypical Alexander disease with dystonia, retinopathy, and a brain mass mimicking astrocytoma. Neurol Genet. 2018;4(4):e248.

11 Hagemann TL, Paylor R, Messing A. Deficits in adult neurogenesis, contextual fear conditioning, and spatial learning in a Gfap mutant mouse model of Alexander disease. J Neurosci. 2013;33(47):18698-706. 